\title{
UNIVERSITYOF
}

FORWARD

THINKING

WESTMINSTER ${ }^{\text {用 }}$

WestminsterResearch

http://www.westminster.ac.uk/westminsterresearch

\section{On Random Sampling and Fourier Transform Estimation in Sea Waves Prediction}

Al-Ani, M., Belmont, M, Christmas, J, Tarczynski, A. and Ahmad, B. I.

This is a copy of the author's accepted version of a paper subsequently published in the proceedings of 2020 Event-Based Control, Communication and Signal Processing. Krakow, Poland, 23 - 25 Sep 2020, IEEE.

The final published version will be available online at:

https://doi.org/10.1109/EBCCSP51266.2020.9291348

(C) 2020 IEEE . Personal use of this material is permitted. Permission from IEEE must be obtained for all other uses, in any current or future media, including reprinting/republishing this material for advertising or promotional purposes, creating new collective works, for resale or redistribution to servers or lists, or reuse of any copyrighted component of this work in other works.

The WestminsterResearch online digital archive at the University of Westminster aims to make the research output of the University available to a wider audience. Copyright and Moral Rights remain with the authors and/or copyright owners. 


\section{On Random Sampling and Fourier Transform Estimation in Sea Waves Prediction}

\author{
Mustafa Al-Ani \\ College of Engineering, Mathematics and \\ Physical Sciences, \\ University of Exeter, \\ Exeter, UK \\ m.t.a.al-ani@exeter.ac.uk
}

\author{
Michael Belmont \\ College of Engineering, Mathematics and \\ Physical Sciences, \\ University of Exeter, \\ Exeter, $U K$ \\ m.r.belmont@exeter.ac.uk
}

\author{
Jacqueline Christmas \\ College of Engineering, Mathematics and \\ Physical Sciences, \\ University of Exeter, \\ Exeter, UK \\ j.t.christmas@exeter.ac.uk
}

\author{
Andrzej Tarczynski \\ College of Computer Science \\ and Engineering, \\ University of Westminster, \\ London, UK \\ tarczya@wmin.ac.uk
}

\author{
Bashar I. Ahmad \\ School of Technology \\ Department of Engineering, \\ University of Cambridge, \\ Cambridge, UK \\ b.i.ahmad@eng.cam.ac.uk
}

\begin{abstract}
Improving the safety of a wide range of launch and recovery operations is of great international maritime interest. Deterministic sea wave prediction (DSWP) is a relatively new branch of science that can offer such opportunities by predicting the actual shape of the sea surface and its evolution for short time in the future. Fourier transform technique is the main building block in DSWP, which requires measurements of the sea surface. Nonetheless, uniformly sampled measurements of the sea surface cannot be practically achieved for various reasons. Conventional $\mathrm{X}$-band radars are the most realistic candidate to provide a low-cost convenient source of two-dimensional wave profile information for DSWP purposes. Ship movement and mechanically rotating scanning antennas are among sources of irregularity in sea surface sampling. This in turn introduces errors when traditional Fourier transform based wave prediction methods are used. In this paper we show that by modelling the radar sampling instants as random variables and using the estimator of Tarczynski and Allay to process the samples, a reliable solution for DSWP can be constituted.
\end{abstract}

Keywords- DSWP, Fourier transform, random sampling, smeared data

\section{INTRODUCTION}

Deterministic Sea Wave Prediction (DSWP) is a relatively new maritime technology which aims to predict the actual shape of the sea surface and its evolution in a location of interest, around a vessel for example, typically in the timescale of several tens of seconds ahead [1], [2]. This new technology has a few applications threads which already have very significant client interest. Mainly, it can extend the sea state at which maritime operations can be carried out safely. These include: (a) the launch and recovery of helicopters, small surface craft and remotely controlled submersibles to mother ships, (b) cargo and personnel transfers. DSWP can achieve that by identifying quiescent periods that are known to exist in large seas where runs of consecutive waves occur with amplitudes significantly less than the mean [3], [4].

DSWP takes measurements of the sea surface profile, construct a linear spectral wave model, and then propagates the wave model in time to the location of interest, e.g. the vessel's site. The spectral coefficients in the linear model are obtained by determining the Fourier transform over the measurements (spatial and temporal) window. Satellite wave measurements cannot provide either the required resolution or coverage, leaving the available sensor technologies such as: wave sensor buoys, scanning wave-profiling LIDAR, or ship mounted wave radar. Wave buoys are not practical for ships and moving vessels, although they do have a research role and can provide accurate representation of the wave profile. Furthermore, wave buoys provide time series of the wave profiles at a single point, which are not enough to describe highly two dimensional seas. Scanning wave-profiling LIDAR systems have the necessary functionality, but they are still at infancy, and the commercially available versions have a short range for DSWP purposes. So for suitable range, convenience and cost, X-band navigation radars are the most attractive sensing technology for DSWP. The typical wave radar data is captured using a mechanically rotated antenna, and hence the sampling points per scan vary in space and in time by up to the rotation period which is about 23 s. Additionally, as more than one scan can be needed in building a reliable sea model, sea surface waves travel between scans leading to further, yet trackable, variation in the spatial sampling points in the data. The simplest approach is to ignore all the temporal and spatial variation in the collected data and use a classical Fourier transform, which leads to inevitable errors. Other solutions include a least-squares fitting of the data to all the spectral components simultaneously which is computationally prohibitive considering the size of the data and 
the live nature of the application. In [1], we fit the data to pairs of sinusoidal components in a one-by-one manner and showed very promising results when applied to a single time-smeared scan. In this paper, we provide an alternative, generalised solution and justification that is based on random sampling estimators. Fourier transform estimation from random sampling fits under the paradigm of Digital Alias-Free Signal Processing (DASP) [5], [6]. The typical objective of these random sampling estimators is to reduce the sampling rates below the required threshold of Nyquist. Generally speaking, these approaches rely on randomising the sampling instants in specific modes and tailor an unbiased Fourier transform estimators to process the samples. Research in this field is usually focused on reducing the estimation error for a given sampling rate [7]-[10]. In this paper, we address a different problem. We show by modelling the radar irregular sampling instants as random variables and process the collected samples using a Fourier transform estimator, we can create a reliable sea wave model which in turn can generate accurate predictions

\section{Sea WaVe Model AND Measurements}

The fundamental linear oceanographic wave model of the sea surface, $h(x, y, t)$, at the coordinates $x, y$, and time $t$ is given by,

with,

$$
h(x, y, t)=\mathcal{R}\left\{\sum_{q=1}^{Q} \sum_{n=1}^{N} c_{n, q} \alpha_{n, q}(x, y, t)\right\}
$$

$$
\begin{array}{r}
\alpha_{n, q}(x, y, t) \triangleq \exp \left(-j\left(k_{n} x \cos \left(\theta_{q}\right)+k_{n} y \sin \left(\theta_{q}\right)-\right.\right. \\
\left.\left.\omega_{n} t\right)\right),(2)
\end{array}
$$

where $c_{n, q}$ is the directional spectral coefficient at the wave number $k_{n}$ and its angular frequency $\omega_{n}$ in the propagation direction $\theta_{q}, N$ is the number of spectral components per direction, $Q$ is the number of wave directions, and $\mathcal{R}\{$.$\} is the$ real part operator. The wave number $k_{n}$ and its angular frequency $\omega_{n}$ are related by the deep water dispersion relationship. The directions of the wave systems $\left\{\theta_{q}\right\}_{q=1}^{Q}$ are set to span $[0,2 \pi)$. The wavenumbers $\left\{k_{n}\right\}_{n=1}^{N}$ are set to uniformly range over the longest and shortest waves to represent. Let $z=$ $(x, y, t)$, the spectral coefficients are obtained through

$$
c_{n, q}=\int_{\mathcal{T}} h(z) \alpha_{n, q}(z) d z
$$

where $\mathcal{T}$ is a window in time and space where/when the wave model (1) can represent the sea surface waves. It is a trivial task to find the coefficients of (1) if an error-free perfect snapshot of the sea surface was available at a signal time instant: a classical Fourier transform techniques (2DFFT for computational efficacy) could be used. However, this is not the case for several reason. First, the sea surface is captured using a radar with a rotating antenna of a period of few seconds. Accordingly, the sampling points vary in space and time. Second, waves travel (at different speeds) during the measurements. Thus, if more than one scan is used in obtaining the coefficients to reduce the

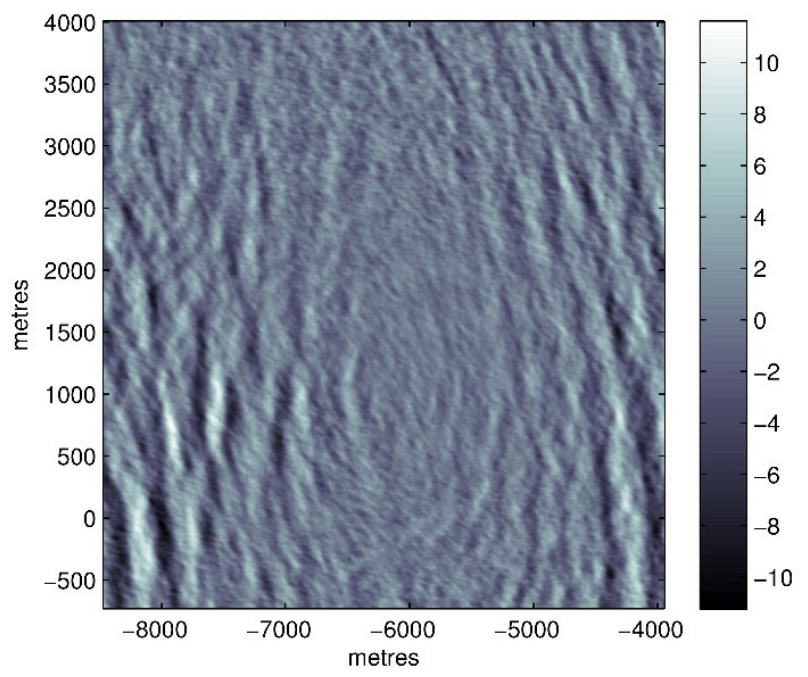

Figure 1: Example wave profile measurement. The shading represents the sea surface elevation, according to the metre scale shown on the right.

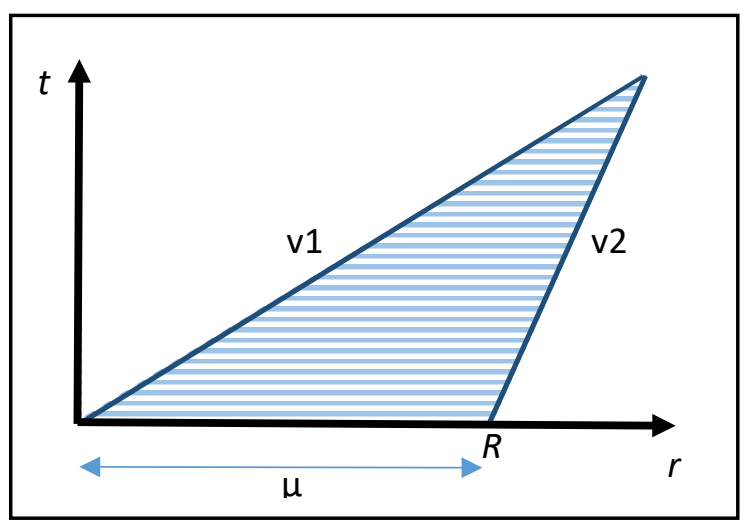

Figure 2: The Space-Time diagram for spatial measurment $\mu$ for waves with fastest velocity v1 and slowest velocity v2. The shadowed triagled area is the prediction zone with lines of slope v1 and v2.

effect of errors in the measurements, the data will be nonuninform in space and time. Additionally ship movement is another source for irregularity. Simply ignore the sampling jitter and treat the radar scan data as a perfect snapshot would constitute a considerable source of error in the prediction as we show in Section IV. Figure 1 shows an example surface profile measured and processed by a commercial wave profiling system during the Golden Arrow sea trial undertaken by the University of Exeter and supported by the Ministry of Defense. The shading indicates the surface elevation, in metres, as given by the colourbar on the right. We can see that the sea surface image suffers from errors.

It is practical and computationally efficient to choose a subdomain from each radar scan, where the measurements are most reliable. The dimensions and the relative location of the reliable measurement zone is determined by few factors: the 


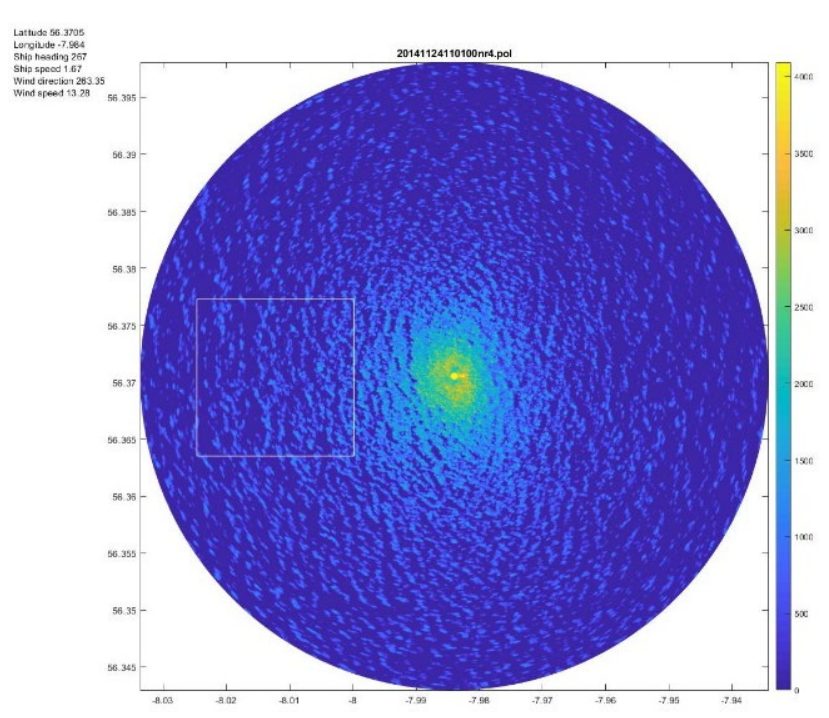

Figure 3. An example of a radar measurement zone from the Golden Arrow sea trial.

prevailing sea condition, radar height and characteristics, and the application requirements. It is usually located in the directions of the waves traveling from towards the radar. Based on these measurements, the built wave model in turn will be used to predict the sea surface profile at future location in time and space, referred to by the prediction zone. The location and dimensions of the prediction zone for a prediction horizon is determined by the Space-Time diagrams [11]. These diagrams yield all the necessary information about the relationship between the properties of the measurement process, the corresponding prediction zone, and available prediction horizon. These diagrams and their extensions show that there is always a user defined compromise to be achieved between prediction zone/horizon and accuracy. The essential features of this information propagation process are shown in Figure 2 of a single polychromatic wave systems along the radar range $r$ ( $r=0$ is where waves are coming from). For the case of twodimensional linear seas, the worst case parameters are extracted from sets of such diagrams taken over the dominant wave directions prevailing at the time. In Figure 3, we show an example of the measurement zone from the Golden Arrow sea trial, where the waves were mainly travelling from the west towards the ship. The ship/radar is in the centre, and the measurement zone is the square on its left.

\section{RANDOM SAMPLING ESTIMATION}

From the radar measurements, we assume $M$ sequence of temporal-spatial sampling points $\left\{z_{m}\right\}_{m=1}^{M}$, where $z_{m}=$ $\left(x_{m}, y_{m}, t_{m}\right)$ are available to us along with the data samples of the sea elevation $\left\{h\left(z_{m}\right)\right\}_{m=1}^{M}$. To obtain the spectral coefficient $c_{n, q}$ in (1), we assume that $\left\{z_{m}\right\}_{m=1}^{M}$ are i.i.d random variables with $p_{n, q}(z)$ distribution function over the measurement zone $\mathcal{T}$, and construct a Fourier transform like estimator as:

$$
\hat{c}_{n, q}=\frac{1}{M} \sum_{m=1}^{M} \frac{\alpha_{n, q}\left(\mathrm{z}_{m}\right) h_{s}\left(\mathrm{z}_{m}\right)}{p_{n, q}\left(z_{m}\right)} .
$$

We note that the expectation of the estimator (4) is identical with the target (2):

$$
\begin{aligned}
E\left[\hat{c}_{n, q}\right] & =\frac{1}{M} \sum_{m=1}^{M} \int_{\mathcal{T}} \alpha_{n, q}(z) h(z) d z, \\
& =\int_{\mathcal{T}} h(z) \alpha_{n, q}(z) d z=c_{n, q},
\end{aligned}
$$

where $E[$.$] is the expectation operator. Thus, p_{n, q}(z)$ must be determined to achieve (5), and hence $\hat{c}_{n, r}$ becomes an unbiased estimator of $c_{n, q}$. As wave components travel at different speeds, $p_{n, q}(z)$ is a wavenumber-dependent function and it can be configured over the measurement window in each wave direction in polar coordinates as follows. Let $r \in(0, R]$ be the radial range of the measurement zone where $r=0$ represents the point in the scan where new waves are coming from, and $R$ is the radial length of the zone, in the direction $\varphi$. The function depends on the number of scans $S$ used in (4) and follows a staircase function (a function composed of a set of equally spaced jumps of equal length) in each direction over the range $r \in\left(0, S D_{n}\right]$, where $D_{n}=\omega_{n} / 2 k_{n}$ is the distance travelled by the waves at wave component at $\left(\omega_{n}, k_{n}\right)$ : $p_{n, q}(r, \varphi)=\left\lceil r / D_{n}\right\rceil / S\|\mathcal{T}\|$, and $p_{n, q}(r, \varphi)=1 / S\|\mathcal{T}\|$ for $\left(S D_{n}, R\right]$, where $\|\mathcal{T}\|$ is the size of the observation window. If a single scan is used, and the ship is stationary, $p_{n, q}(r, \varphi)$ will be a uniform function of value $1 /\|\mathcal{T}\|$.

\section{NUMERICAL RESULTS}

The aim of this section is to compare the prediction results of the estimator (4) with the traditional two-dimensional Fourier transform based method where the data is assumed/approximated to be perfectly captured with no temporal variation. For simple illustration of the capability of (4) at handling the unevenly collected data, we use one radar scan with varying rotation scanning time. The ship is assumed stationary. The comparison is conducted using numerical simulations of various sea states. JONSWAP spectra are used here to simulate the sea surface with peakedness parameter of 3.3 and a spread function of 12 in the original Cartwright form leading to directional spectra spread under one half plane. The standard linear method of adding a large number of sinusoidal components is used to generate the data. The phases were generated from a uniform random distribution over $[0,2 \pi)$ and in order to obtain waves with the correct randomness, the amplitudes of the components were obtained by sampling from a frequency dependent Rayleigh distribution [12]. The variance of the Rayleigh distribution at each frequency was obtained from the spread JONSWAP power density spectra.

The sea states are described in terms of the wave spectral parameters: the significant wave height $\mathcal{H}$ and mean period $\mathcal{P}$ in the first columns of Table 1 and 2 . The radar rotation period $\Delta t$ is set to 2 and $3 \mathrm{~s}$. The simulated data covers a $2 \mathrm{~km}$ radius 
circle with an angular resolution of $0.5 \mathrm{deg}$ and range resolution of $3 \mathrm{~m}$. The simulated radar measurements vary in time and space. However, no other sources of error such as shadowing and measurement noise are introduced to the synthetic data.

Each Experiment was run for 1 hour, predicting the wave profile for prediction horizon of $[0,120] \mathrm{s}$, every minute. The correlation and the normalized error between the predicted and the actual wave profiles are determined. For each experiment the sea state is stationary and therefore it was deemed sufficient to depict the results in terms of the mean correlation $A$ and the mean normalised RMS error $C$ in Table 1 and 2. Under all the sea conditions the random sampling estimators using the correct temporal and spatial sampling locations performs significantly better than the traditional two-dimensional Fourier approach that assume a spatial snapshot of the surface not accounting for the time variation. The approximation approach is worse for longer radar rotation period and shorter wave periods as expected because of the worsen snapshot approximation. However, there is no effect on the random sampling estimator.

Table 1. The performance of the traditional Fourier technique.

\begin{tabular}{c|c|c}
\hline \multirow{3}{*}{ Sea State } & $\Delta t=2 \mathrm{~s}$ & $\Delta t=3 \mathrm{~s}$ \\
& & \\
& $C$, & $C$, \\
\hline $\mathcal{H}=4.7 \mathrm{~m}$ & $\mathbf{0 . 9 1 ,}$ & $\mathbf{0 . 7 8 ,}$ \\
$\mathcal{P}=8.7 \mathrm{sec}$ & 0.41 & 0.61 \\
\hline $\mathcal{H}=6 \mathrm{~m}$ & $\mathbf{0 . 9 2 ,}$ & $\mathbf{0 . 8 6 ,}$ \\
$\mathcal{P}=9.4 \mathrm{sec}$ & 0.39 & 0.52 \\
\hline $\mathcal{H}=6.7 \mathrm{~m}$ & $\mathbf{0 . 9 4}$, & $\mathbf{0 . 8 5}$, \\
$\mathcal{P}=9.7 \mathrm{sec}$ & 0.33 & 0.55 \\
\hline $\mathcal{H}=7.3 \mathrm{~m}$ & $\mathbf{0 . 9 4 ,}$ & $\mathbf{0 . 8 3}$, \\
$\mathcal{P}=10 \mathrm{sec}$ & 0.34 & 0.57 \\
\hline
\end{tabular}

Table 2. The performance of the random sampling estimator.

\begin{tabular}{c|c|c}
\hline & $\Delta t=2 \mathrm{~s}$ & $\Delta t=3 \mathrm{~s}$ \\
Sea State & $A$, & $A$, \\
& $C$ & $C$ \\
\hline \hline $\mathcal{H}=4.7 \mathrm{~m}$ & $\mathbf{0 . 9 9}$ & $\mathbf{0 . 9 8}$, \\
$\mathcal{P}=8.7 \mathrm{sec}$ & 0.11 & 0.13 \\
\hline $\mathcal{H}=6 \mathrm{~m}$ & $\mathbf{0 . 9 9}$, & $\mathbf{0 . 9 9}$, \\
$\mathcal{P}=9.4 \mathrm{sec}$ & 0.10 & 0.08 \\
\hline $\mathcal{H}=6.7 \mathrm{~m}$ & $\mathbf{0 . 9 9}$, & $\mathbf{0 . 9 9}$, \\
$\mathcal{P}=9.7 \mathrm{sec}$ & 0.12 & 0.11 \\
\hline $\mathcal{H}=7.3 \mathrm{~m}$ & $\mathbf{0 . 9 9}$, & $\mathbf{0 . 9 9}$, \\
$\mathcal{P}=10 \mathrm{sec}$ & 0.08 & 0.13 \\
\hline
\end{tabular}

\section{REFERENCES}

[1] M. Al-Ani, J. Christmas, M.R. Belmont, J.M. Duncan, J. Duncan, and B. Ferrier, "Deterministic Sea Waves Prediction Using Mixed Space-Time Wave Radar Data," J. of Atmos. Oceanic Technol., vol. 36, pp. 833-842, 2019.

[2] B. Ferrier, A. Baitis and A. Manning, 2000: Evolution of the landing period designator (LPD) for shipboard air operations. Nav. Eng. J., 112 (4), 297-316.

[3] P. Crossland, J.M. Duncan, and B. Ferrier, 2009: The feasibility of developing a quiescent period prediction system in a simulation environment. Proc. of the Int. Conf. on Computer Applications in Shipbuilding (ICCAS), Shanghai, China, 2. 751762 .

[4] M. Al-Ani, M.R. Belmont, and J. Christmas, "On Numerically Obtaining the Probability Distribution of Quiescent Periods from Wave Power Spectral Density", IEEE Trans. On Ocean Engineering, 2020.

[5] Bilinskis, Digital Alias-free Signal Processing. UK: John Wiley \& Sons, 2007.

[6] I. Bilinskis and A. Mikelsons, Randomized Signal Processing. Englewood Cliffs, NJ, USA: Prentice-Hall, 1992.

[7] A. Tarczynski and N. Allay, "Spectral analysis of randomly sampled signals: suppression of aliasing and sampler jitter," IEEE Trans. on Signal Processing, vol. SP-52, pp. 3324-3334, 2004.

[8] M. Al-Ani and A. Tarczynski, "Evaluation of Fourier transform estimation schemes of multidimensional signals using random sampling," Signal processing, vol. 92, pp. 2484-2496, 2012.

[9] M. Al-Ani and A. Tarczynski, "Efficient multidimensional sampling scheme for Fourier transform estimation," in Proc. of the IEEE Int. Conf. on Circuits and Systems (ISCAS), Seoul, 2012, pp. 3210-3213.

[10] M. Al-Ani, A. Tarczynski and B. I. Ahmad, "A Novel Fourier transform estimation method using random sampling," in Proc. of the 19th European Signal Processing Conference (EUSIPCO'11), Aug. 2011, pp. 859-863.

[11] Abusedra, L., and M. Belmont, 2011: Prediction diagrams for deterministic sea wave prediction and the introduction of the data extension prediction method. Int. J. Shipbuild. Prog., 58, 59-81.

[12] M. J. Tucker, P. G. Challenor, and D. J. T. Carter, "Numerical simulation of a random sea: A common error and its effect upon wave group statistics," Appl. Ocean Res., vol. 6, pp. 118 $122,1984$. 